Louisiana State University

LSU Digital Commons

Faculty Publications

Department of Physics \& Astronomy

$10-14-2014$

\title{
Amplification of angular rotations using weak measurements
}

Omar S. Magaña-Loaiza

University of Rochester Institute of Optics

Mohammad Mirhosseini

University of Rochester Institute of Optics

Brandon Rodenburg

University of Rochester Institute of Optics

Robert W. Boyd

University of Rochester Institute of Optics

Follow this and additional works at: https://digitalcommons.Isu.edu/physics_astronomy_pubs

\section{Recommended Citation}

Magaña-Loaiza, O., Mirhosseini, M., Rodenburg, B., \& Boyd, R. (2014). Amplification of angular rotations using weak measurements. Frontiers in Optics, FiO 2014 https://doi.org/10.1364/fio.2014.fth4a.4

This Conference Proceeding is brought to you for free and open access by the Department of Physics \& Astronomy at LSU Digital Commons. It has been accepted for inclusion in Faculty Publications by an authorized administrator of LSU Digital Commons. For more information, please contact ir@lsu.edu. 


\title{
Amplification of Angular Rotations Using Weak Measurements
}

\author{
Omar S. Magaña-Loaiza, ${ }^{1, *}$ Mohammad Mirhosseini, ${ }^{1, \dagger}$ Brandon Rodenburg, ${ }^{1}$ and Robert W. Boyd ${ }^{1,2}$ \\ ${ }^{1}$ The Institute of Optics, University of Rochester, Rochester, New York 14627, USA \\ ${ }^{2}$ Department of Physics, University of Ottawa, Ottawa, Ontario K1N 6N5, Canada
}

(Received 10 February 2014; published 21 May 2014)

\begin{abstract}
We present a weak measurement protocol that permits a sensitive estimation of angular rotations based on the concept of weak-value amplification. The shift in the state of a pointer, in both angular position and the conjugate orbital angular momentum bases, is used to estimate angular rotations. This is done by an amplification of both the real and imaginary parts of the weak-value of a polarization operator that has been coupled to the pointer, which is a spatial mode, via a spin-orbit coupling. Our experiment demonstrates the first realization of weak-value amplification in the azimuthal degree of freedom. We have achieved effective amplification factors as large as 100, providing a sensitivity that is on par with more complicated methods that employ quantum states of light or extremely large values of orbital angular momentum.
\end{abstract}

DOI: 10.1103/PhysRevLett.112.200401

PACS numbers: 03.65.Ta, 42.25.Hz, 42.50.Ex, 42.50.Tx

In 1988, Aharonov et al. [1] introduced a general form of quantum measurement, known as a weak measurement. In weak measurements, information is gained by weakly probing the system, while approximately preserving its initial state. The uncertainty in each measurement is large due to the weak perturbative nature of the information extraction; however, this is generally overcome by averaging over a large number of identically prepared states. The process of postselecting the prepared system makes weak measurements interesting. Under certain conditions, the outcome, which is called a weak value (WV), is not an eigenvalue of the measurement operator. In fact, WVs can even exceed the eigenvalue range of a typical strong or projective measurement and, in general, are complex. These features have allowed a wide range of applicability in classical and quantum contexts. For example, they have resulted in the measurement via amplification of small transverse [2,3] and longitudinal [4-6] shifts, the direct measurement of the quantum wave function [7-9], the development of tomographic techniques [10], the amplification of optical nonlinearities [11], and the clarification of controversial debates in quantum physics $[12,13]$.

Recently, there has been a strong impetus to employ weak-value amplification (WVA) as an effective tool in metrology $[4,5,14,15]$. A WVA protocol involves the preparation of an ensemble of particles with two independent degrees of freedom (DOFs). These two DOFs are then coupled by means of a weak perturbation and postselected to collapse one of the DOFs, typically called the probe. Because of the coupling existing between the probe and the other DOF, called the pointer, the postselection induces a shift in the linear position of the pointer which is proportional to the weakly induced perturbation and the WV. This has allowed the use of WVA to estimate small quantities with sensitivities comparable to quantum-enhanced metrology $[4,5,14-16]$, due to the fact that the use of quantum protocols does not guarantee sensitivities beyond the standard quantum limit, which is the limit for classical protocols $[17,18]$.

Besides the extensive work on the estimation of longitudinal displacements $[4-6,14,17-19]$, high sensitivity measurement of angular displacements has been another topic of interest. Historically, inquiries regarding relativistic dynamics stimulated interest in the azimuthal DOF [20]. A remarkable example is the Sagnac effect. Atomic versions of the Sagnac interferometer have led to sensitive gyroscopes that permit a precise measurement of rotations [21,22]. In addition, the use of light endowed with orbital angular momentum (OAM) has motivated interest in new forms of rotations. As identified by Allen et al. [23], an optical beam with azimuthal phase dependence of the form $e^{i \ell \phi}$ carries OAM, where $\phi$ is the azimuthal angle and $\ell$ is the OAM value. These beams have been used for rotational control of microscopic systems [24], and exploration of effects such as the rotational Doppler shift [25] which has been recently used in techniques for detecting spinning objects [26,27]. Recent efforts to increase the sensitivity in the measurement of angular rotations involve the generation of large values of OAM [28], quantum entanglement of high OAM values [29], or the use of NOON states in the OAM bases [30]. These protocols require complicated schemes to generate and measure photons in such exotic states. However, the concepts behind them constitute valuable resources not only for optical metrology, remote sensing, biological imaging, or navigation systems [26,27,31], but also for the understanding of light-matter interactions [32-34].

In this Letter, we describe WVA in the azimuthal DOF and the processes that give rise to these effects. The first observation of these kinds of WVs suggests interesting physics from the fundamental and applied perspective. For instance, the spinorbit coupling in our experiment gives rise to an interesting optical effect in which the perturbation of polarization induces a shift in the angular position and OAM spectrum of the pointer. 
We show that the real and the imaginary part of the WV for the polarization operator can be accessed by measuring the angular position and its conjugate variable of OAM, respectively. Using this new form of WVs based on spin-orbit coupling, we propose a scheme for the measurement of small rotations. We demonstrate an amplification in the measurement of angular rotations that is as large as 100 . The simplicity of our scheme, namely the lack of need for an exotic quantum state of light or extremely large values of OAM, makes this technique potentially attractive for applications in optical metrology, remote sensing, and optical manipulation of microscopic systems.

Consider the experimental setup depicted in Fig. 1. This scheme comprises three parts: state preparation, a weak perturbation, and postselection. The state preparation involves the generation of a light beam with diagonal polarization and a well-defined spatial profile. We select the initial polarization state using a polarizer and a half-wave plate (HWP); this state will serve as a probe and can be described by the polarization qubit $\left|\Psi_{\mathrm{pr}}\right\rangle=1 / \sqrt{2}(|H\rangle+|V\rangle)$. The preparation of the spatial mode or pointer consists of the generation of an angular mode (ANG) $f(\phi) \propto \exp \left(-\phi^{2} / 2 \eta_{\phi}{ }^{2}\right)$, which is a Gaussian-apodized angular slit of width $\eta_{\phi}$. This is shaped by impressing amplitude and phase information onto the beam by means of modulation of the blaze parameters on a spatial light modulator (SLM), used together with a $4 f$ optical system containing a spatial filter in the Fourier plane [35]. The advantages that pointer states carrying OAM provide over Gaussian pointer states have been studied [36]. The beam is injected into a Sagnac interferometer, where the horizontally and vertically polarized components of the beam circulate in opposite directions. The Dove prism (DP) is rotated by a small angle $\Delta \phi / 4$ with respect to the plane of the interferometer, which causes the two counterpropagating beams to be rotated by an amount of $\pm \Delta \phi / 2$ in opposite directions. This setup enables a coupling between the polarization, marked by the two counterpropagating beams, and the transverse azimuthal DOF. In the next step, we use two quarter-wave plates (QWPs) and a HWP to induce a geometric phase between the two circulating beams in the interferometer, permitting the existence of complex WVs (see the Supplemental Material [37]). Finally, the postselection is carried out by setting the angle of a polarizer almost orthogonal with respect to the angle of the polarizer used in the preselection. At this stage, a full characterization of the complex wave function in the transverse angular basis and the conjugate basis of OAM reveals information about the real and the imaginary part of the $\mathrm{WV}$, respectively.

The interaction in our experiment can be described by the spin-orbit interaction Hamiltonian $\hat{H}_{\mathrm{SO}}=\mu \hat{\sigma} \hat{\ell}_{z}$ and a Hamiltonian that describes the action of the wave plates $\hat{H}_{g}=\delta \hat{\sigma}$, where $\hat{\sigma}$ is the Pauli operator defined by $\hat{\sigma} \equiv|H\rangle\langle H|-| V\rangle\langle V|, \Delta \phi / 2=\mu \Delta t,\left(\theta_{H} / 2-\pi / 2\right)=\delta \Delta t$, and $\theta_{H} / 2$ is the induced geometric phase. Our state at the input of the interferometer has the following form $\left|\Psi_{i}\right\rangle=\left|\Psi_{\text {pr }}\right\rangle|f(\phi)\rangle$. The interaction which occurs in the interferometer couples the two DOFs as follows:

$$
\begin{aligned}
\left|\Psi_{f}\right\rangle & =e^{-i \frac{\Delta \phi}{2} \hat{\sigma} \hat{\theta}_{z}} e^{-i \hat{\sigma}\left(\frac{\theta_{H}}{2}-\frac{\pi}{2}\right)}\left|\Psi_{i}\right\rangle \\
& =\frac{1}{\sqrt{2}}\left(e^{-i \frac{\theta}{2}}|H\rangle|f(\phi-\Delta \phi / 2)\rangle+e^{i \frac{\theta}{2}}|V\rangle|f(\phi+\Delta \phi / 2)\rangle\right),
\end{aligned}
$$

where $\left(\hat{\ell}_{z}\right)$ act as the generator of rotations and is proportional to the angular momentum operator projected along the optical axis $\hat{L}_{z}=\hbar \hat{\ell}_{z}$, and $\theta$ equals $\theta_{H}-\pi$. As demonstrated by Eq. (1), the weak coupling creates entanglement between probe and pointer. It should be noted that since the probe and the pointer are different DOFs of a single beam rather than separate systems or particles, then this is an example of classical entanglement and, thus, can be described classically $[38,39]$. Because of this, most traditional weak measurement experiments, such as those described in Refs. [2,4,5,7-10,36], are classically explainable. This also demonstrates what is required to perform a nonclassical weak measurement experiment. We have

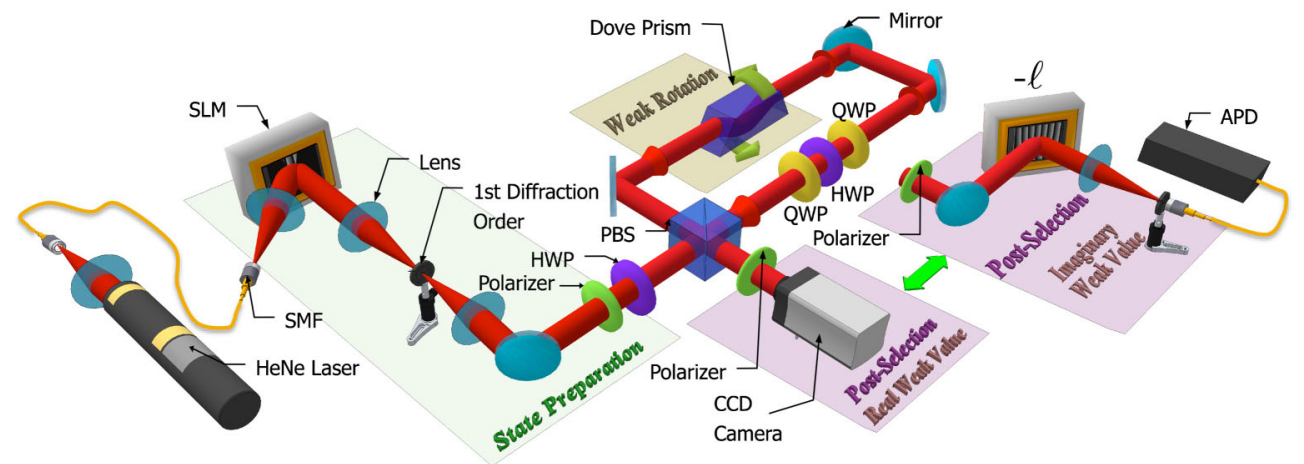

FIG. 1 (color online). Experimental setup. A light beam from HeNe laser working at $632.8 \mathrm{~nm}$ is coupled into a SMF and the output is then collimated. The beam is sent to a phase-only SLM and then to a $4 f$ optical system containing a spatial filter in the Fourier plane. A polarization state is prepared by means of a polarizer and a HWP. A Dove prism, a HWP and two QWPs are placed inside the Sagnac interferometer that uses a polarizing beam splitter (PBS). The DP induces a small rotation between the counterpropagating beams; this is the weak perturbation. The QWPs together with the HWP induce a geometric phase between the $H$ and $V$ polarized beams. After postselection, measurements of angular rotations and OAM spectra are performed to access the real or imaginary part of the weak value. 
chosen to use the mature language of weak measurement theory, since it provides a simpler description and the results readily apply to a wider range of phenomena including nonclassical systems.

The postselection is performed by projecting the perturbed state into $\left|\Phi_{\mathrm{ps}}\right\rangle=\sin (\gamma / 2-\pi / 4)|H\rangle+\cos (\gamma / 2-\pi / 4)|V\rangle$, where $\gamma$ is controlled by the polarizer. The postselection collapses the polarization state of the probe and causes a shift in the angular position and the OAM spectrum of the pointer that can be described as

$$
\left|\Psi_{p}\right\rangle=\left|\Phi_{\mathrm{ps}}\right\rangle\left\langle\Phi_{\mathrm{ps}} \mid \Psi_{f}\right\rangle \approx\left|\Phi_{\mathrm{ps}}\right\rangle\left|f\left(\phi-\sigma_{w} \Delta \phi / 2\right)\right\rangle .
$$

Here, $\sigma_{w}$ is the complex WV given by

$$
\sigma_{w} \equiv \frac{\left\langle\Phi_{\mathrm{ps}}|\hat{\sigma}| \Psi_{\mathrm{fpr}}\right\rangle}{\left\langle\Phi_{\mathrm{ps}} \mid \Psi_{\mathrm{fpr}}\right\rangle}
$$

$\left|\Psi_{\text {fpr }}\right\rangle$ is defined as $1 / \sqrt{2}\left(e^{-i(\theta / 2)}|H\rangle+e^{i(\theta / 2)}|V\rangle\right)$. If the induced phase $\theta$ and polarizer selection angle $\gamma / 2$ are small, the WV can be approximated as (see the Supplemental Material [40])

$$
\sigma_{w} \approx-\frac{2 \gamma}{\gamma^{2}+\theta^{2}}+i \frac{2 \theta}{\gamma^{2}+\theta^{2}}
$$

The postselected state described in Eq. (2) shows a change in angle as $\phi \rightarrow \phi-\sigma_{w} \Delta \phi / 2$. If $\sigma_{w}$ is real, which will be the case for $\theta=0$, then this leads to the rotation of the pointer by the amount $\sigma_{w}$. However, if $\sigma_{w}$ is complex then

$$
\begin{aligned}
f\left(\phi-\sigma_{w} \Delta \phi / 2\right) & =e^{\left(-\left(\phi-\sigma_{w} \Delta \phi / 2\right)^{2} / 2 \eta_{\phi}^{2}\right)} \\
& \propto e^{\left(-\left[\phi-\Re\left(\sigma_{w}\right) \Delta \phi / 2\right]^{2} / 2 \eta_{\phi}^{2}\right)} e^{\left(i \phi \Im\left(\sigma_{w}\right) \Delta \phi / 2 \eta_{\phi}^{2}\right)} \\
& =e^{\left(-(\phi-\Delta\langle\phi\rangle)^{2} / 2 \eta_{\phi}^{2}\right)} e^{(i \phi \Delta\langle\ell\rangle)},
\end{aligned}
$$

where $\Delta\langle\phi\rangle=\Re\left(\sigma_{w}\right) \Delta \phi / 2$ sets the amount of the pointer's rotation. In addition, the pointer experiences a shift in its OAM spectrum that equals $\Delta\langle\ell\rangle=\Im\left(\sigma_{w}\right) \Delta \phi / 2 \eta_{\phi}^{2}$. We have used the angular representation of the spatial mode of the photons, and utilized the Fourier relation between the conjugate pairs of azimuthal angle and angular momentum. Alternatively, the same results can be derived by using the commutation relation between angular position and OAM operators, which is given by $\left[\hat{\phi}, \hat{L}_{z}\right]=i \hbar[1-2 \pi P(\phi)]$, where $P(\phi)$ represents the angular probability at the boundary of the angle range [41]. The shift in the OAM spectrum can be understood as a form of interaction between spin angular momentum (SAM) and OAM. This interesting optical effect is a consequence of the polarization-sensitive nature of the interactions in the interferometer, and should not be confused with the standard spin-orbit coupling in the vector beams where both the SAM and OAM are directed along the same axis [42].

In the experiment, we use a $3 \mathrm{~mW}$ He-Ne laser $(632.8 \mathrm{~nm})$ which is coupled to a single-mode fiber (SMF) and then expanded to a spot size of $1.8 \mathrm{~cm}$. The central part of the beam homogeneously illuminates the display of the SLM that has an active area of $\left(9.3 \times 7 \mathrm{~mm}^{2}\right)$. Because of the reflectance of the SLM and the efficiency of the encoded diffractive grating on it, the power drops to $470 \mathrm{nW}$ once an ANG mode of width $\eta_{\phi}=13.7^{\circ}$ is generated. The DP in the Sagnac interferometer is rotated by $0.3^{\circ}$, this angle is determined by measuring a relative rotation of $1.2^{\circ}$ between two identical ANG modes propagating in the opposite directions. The induced displacement $\Delta \phi$, is chosen to be much smaller than the width of the ANG mode in order to guarantee the conditions for the weak perturbation. The postselection polarizer is set to an angle $\gamma / 2$, with respect to the polarization state of the preselected state. For this part, we have set $\theta$ to zero.

Since our interest is in the amplification of the weak value, the angle $\gamma / 2$ is set to a small number. The postselection polarizer forces the two ANG modes to coherently interfere, producing another ANG mode which is rotated due to the azimuthal Gaussian intensity distribution impressed on the ANG [43]. Such rotation is proportional to the angular displacement $\Delta \phi$ and the real part of the WV $\Re\left(\sigma_{w}\right)$. Since the WV can take values larger than one, this scheme allows the amplification of small rotations. However, as $\Re\left(\sigma_{w}\right)$ is increased more photons are lost as shown for different postselection angles in Figs. 2(a)-2(e). In order to detect this effect, a CCD camera is placed after the polarizer. This is equivalent to measuring the expected value of the angular position in the state $\left|\Psi_{p}\right\rangle$. As shown in Figs. 2(f)-2(j), the measured power is in the range of $10-30 \mathrm{nW}$; however, these images were taken using long exposure times. As can be seen in Fig. 2, an aggressive postselection leads to a larger rotation. The amplification factor (Amp) is defined as the ratio between the angular position of the postselected mode $\Delta\langle\phi\rangle$ and $\Delta \phi$. This is equal to $\Re\left(\sigma_{w}\right) / 2$. Both $\Delta\langle\phi\rangle$ and $\Delta \phi$ were determined by using centroid measurements. The amplification limit is given by the extinction ratio of the polarizer and the magnitude of the weak perturbation or the angle of postselection. Larger amplifications can be measured if the width of ANG is increased and the postselection angle is decreased.

The imaginary part of the WV can be determined by analyzing the shift of the OAM spectrum of the ANG. We have chosen the rotation angle of the DP to be approximately $0.4^{\circ}$, and we have tried different angular widths for the input state. In order to allow $\Im\left(\sigma_{w}\right)$ to be nonzero, the phase $\theta$ must also be nonzero. This is done by inducing a geometric phase between the polarization states $|H\rangle$ and $|V\rangle$. This phase is created using three rotatable wave plates as shown in Fig. 1. The angle of the QWPs is set to $\pi / 4$ and the HWP is rotated by a small angle (see the Supplemental Material [37]). We have set the HWP to an angle such that $\theta / 2=5^{\circ}$ and tried several different postselection angles for the polarizer. Measurement of the OAM spectrum associated with a beam can be done using a wide variety of techniques [44-47]. We measured the OAM using a series of projective measurements for various values of $\ell$. Using a similar procedure as that used for generating the angular slits, a hologram was impressed onto a SLM and then a Fourier transforming 


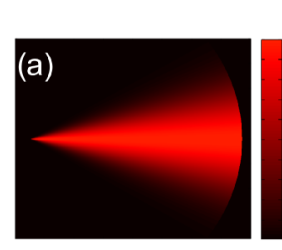

No Post-Selection

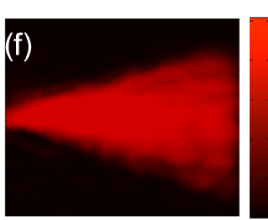

No Post-Selection

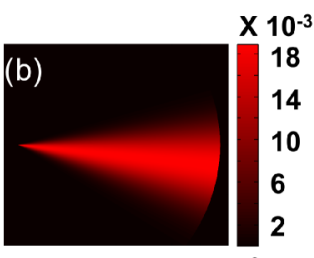

Amp $=4.96 \quad \mathrm{PA}=5^{0}$

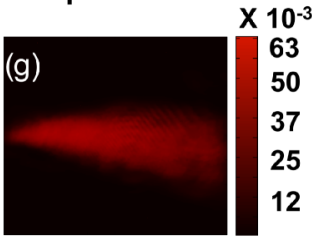

$A m p=4.80 \mathrm{PA}=5^{0}$

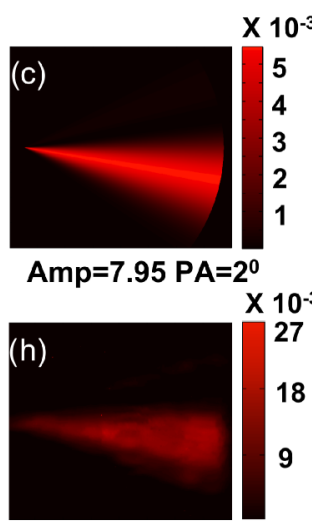

$A m p=7.12 \mathrm{PA}=2^{0}$

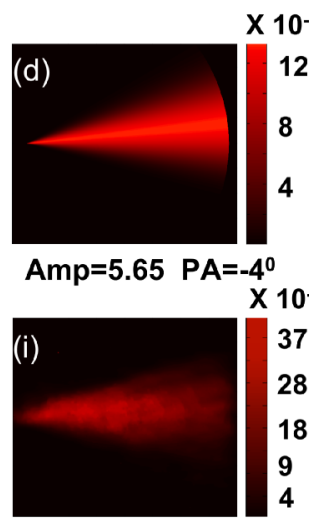

$A m p=5.86$ PA $=-4^{0}$ $\times 10^{-3}$

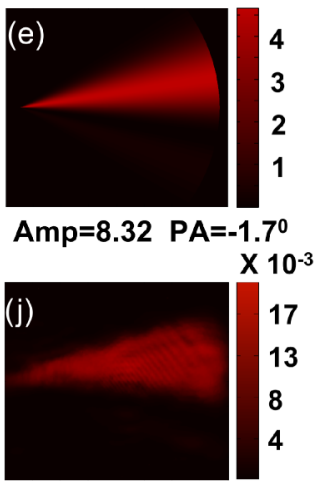

$A m p=8.01$ PA $=-1.7^{0}$

FIG. 2 (color online). Amplification of angular displacements using real weak values. (a)-(e) show simulations of our scheme for $\Delta \phi=1.2^{\circ}$, different postselection angles (PA) and amplification factors (Amp). (f)-(j) show experimental evidence of our protocol under the same conditions.

lens and a spatial filtering from a SMF couples photons to an avalanche photodiode (APD) which allows measurement at single photon levels (see the Supplemental Material [48]).

We summed the counts during a 0.2 second window and averaged it for 30 measurements for each projection over different OAM modes. This procedure was repeated for each mode and the reconstructed spectra are shown in Figs. 3(a)-3(b). The error bars represent the standard deviation over the ensemble of 30 measurements. The spectrum is broader for angular modes with narrower widths due to the uncertainty relation between angular position and OAM [41]. As predicted by Eq. (5), and shown in Fig. 3, the larger amplifications are obtained for angular modes with narrow widths. However, such narrow ANG modes have physically smaller cross sections and, hence, carry proportionally less power. Each OAM power spectrum was fitted using a weighted least-squares minimization to a shifted Gaussian function. The mean values are plotted in Fig. 3(c) along with error bars representing the $3 \sigma$ confidence interval. By exploiting the measurement process, we have amplified small rotations by a magnitude of 100 without using high OAM or entanglement.

Recently, there has been research casting doubt on the sensitivity of measurements based on WVA $[49,50]$. However, it has been shown that in the presence of technical noise WVs, and more specifically imaginary WVs, outperform traditional measurements [51]. Therefore, our experiment can be potentially useful for sensitive measurement of small rotation in real world scenarios. A quantitative analysis of the sensitivity of our scheme can be done by using the Fisher information metric. However, such quantitative comparison is outside the scope of the current work and will be the subject of a future study.

We have made the first step towards the study of WVA in the azimuthal DOF. This has been approached by describing the mechanisms that lead to a shift in the angular position and OAM of an optical beam. The OAM spectrum is shifted as a consequence of the breakup in the polarization symmetry, realized by a differential geometric phase. Furthermore, we have implemented the first realization of
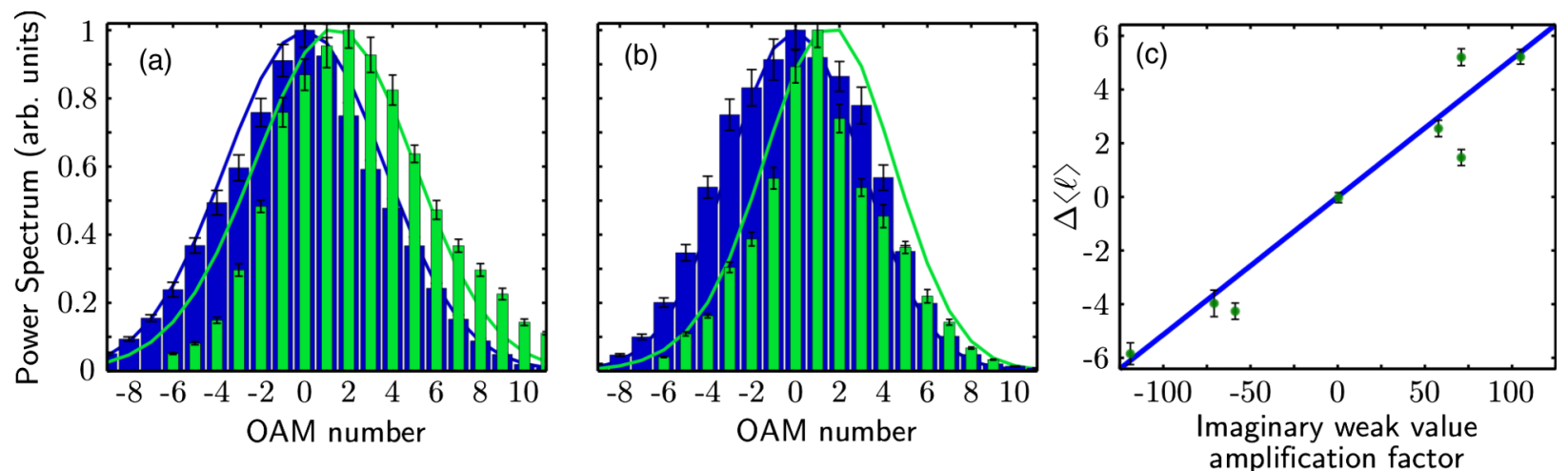

FIG. 3 (color online). Measured OAM power spectra of $\left|\Psi_{p}\right\rangle$ without postselection (blue) and with postselection (green) demonstrating the shift in $\langle\ell\rangle$ due to $\Im\left(\sigma_{w}\right)$ for (a) $\eta_{\phi}=11.4^{\circ}, \gamma / 2=6^{\circ}$ and (b) $\eta_{\phi}=13.7^{\circ}$ and $\gamma / 2=5^{\circ}$. The angle $\theta / 2$ equals $5^{\circ}$ for all the cases. Histograms represent measured data, while lines represent theoretically predicted shifts. (c) OAM centroid shift $\Delta\langle\ell\rangle$ for various measured OAM power spectra plotted against the imaginary WV amplification factor, $\Im\left(\sigma_{w}\right) / 2 \eta_{\phi}^{2}$. Dots represent data, while the line is the theoretical linear curve predicted by Eq. (5). 
WVA in the angular position and OAM bases. The results presented here provide a proof-of-principle demonstration of the scope of WVA in this DOF. We believe that our protocol opens the possibility for new schemes in optical metrology. In addition, our approach shows an alternative fashion for studying the exchange between SAM and OAM in optical systems.

We acknowledge discussions with J. Vornehm, G. Viza, J. Martinez-Rincon, J. C. Howell, and E. Karimi. This work was supported by DARPA, CERC, and CONACyT.

*omar.maganaloaiza@ rochester.edu †mirhosse@optics.rochester.edu

[1] Y. Aharonov, D. Z. Albert, and L. Vaidman, Phys. Rev. Lett. 60, 1351 (1988).

[2] P. B. Dixon, D. J. Starling, A. N. Jordan, and J. C. Howell, Phys. Rev. Lett. 102, 173601 (2009).

[3] O. Hosten and P. Kwiat, Science 319, 787 (2008).

[4] N. Brunner and C. Simon, Phys. Rev. Lett. 105, 010405 (2010).

[5] X.-Y. Xu, Y. Kedem, K. Sun, L. Vaidman, C.-F. Li, and G.-C. Guo, Phys. Rev. Lett. 111, 033604 (2013).

[6] G. Strübi and C. Bruder, Phys. Rev. Lett. 110, 083605 (2013).

[7] J. S. Lundeen, B. Sutherland, A. Patel, C. Stewart, and C. Bamber, Nature (London) 474, 188 (2011).

[8] J. Z. Salvail, M. Agnew, A. S. Johnson, E. Bolduc, J. Leach, and R. W. Boyd, Nat. Photonics 7, 316 (2013).

[9] M. Malik, M. Mirhosseini, M. P. J. Lavery, J. Leach, M. J. Padgett, and R. W. Boyd, Nat. Commun. 5, 4115 (2014).

[10] H. Kobayashi, K. Nonaka, and Y. Shikano, arXiv:1311.3357 [Phys. Rev. D (to be published)].

[11] A. Feizpour, X. Xing, and A. M. Steinberg, Phys. Rev. Lett. 107, 133603 (2011)

[12] S. Kocsis, B. Braverman, S. Ravets, M. J. Stevens, R. P. Mirin, L. K. Shalm, and A. M. Steinberg, Science 332, 1170 (2011).

[13] Y. Aharonov, A. Botero, S. Popescu, and B. Reznik, Phys. Lett. A 301, 130 (2002).

[14] L. J. Salazar-Serrano, D. Janner, N. Brunner, V. Pruneri, and J. P. Torres, Phys. Rev. A 89, 012126 (2014).

[15] H. F. Hofmann, Phys. Rev. A 83, 022106 (2011).

[16] V. Giovannetti, S. Lloyd, and L. Maccone, Nat. Photonics 5, 222 (2011).

[17] N. Thomas-Peter, B. J. Smith, A. Datta, L. Zhang, U. Dorner, and I. A. Walmsley, Phys. Rev. Lett. 107, 113603 (2011).

[18] H. Shin, O. S. Magaña-Loaiza, M. Malik, M. N. O’Sullivan, and R. W. Boyd, Opt. Express 21, 2816 (2013).

[19] B. L. Higgins, D. W. Berry, S. D. Bartlett, H. M. Wiseman, and G. J. Pryde, Nature (London) 450, 393 (2007).

[20] L. H. Thomas, Nature (London) 117, 514 (1926).

[21] T. L. Gustavson, P. Bouyer, and M. A. Kasevich, Phys. Rev. Lett. 78, 2046 (1997).

[22] J. K. Stockton, K. Takase, and M. A. Kasevich, Phys. Rev. Lett. 107, 133001 (2011).

[23] L. Allen, M. W. Beijersbergen, R. J. C. Spreeuw, and J. P. Woerdman, Phys. Rev. A 45, 8185 (1992).

[24] M. Padgett and R. Bowman, Nat. Photonics 5, 343 (2011).
[25] J. Courtial, D. A. Robertson, K. Dholakia, L. Allen, and M. J. Padgett, Phys. Rev. Lett. 81, 4828 (1998).

[26] M. P. J. Lavery, F. C. Speirits, S. M. Barnett, and M. J. Padgett, Science 341, 537 (2013).

[27] C. Rosales-Guzmán, N. Hermosa, A. Belmonte, and J. P. Torres, Sci. Rep. 32815 (2013).

[28] V. D’Ambrosio, N. Spagnolo, L. Del Re, S. Slussarenko, Y. Li, L. C. Kwek, L. Marrucci, S. P. Walborn, L. Aolita, and F. Sciarrino, Nat. Commun. 4, 1 (2013).

[29] R. Fickler, R. Lapkiewicz, W. N. Plick, M. Krenn, C. Schaeff, S. Ramelow, and A. Zeilinger, Science 338, 640 (2012).

[30] A. K. Jha, G. S. Agarwal, and R. W. Boyd, Phys. Rev. A 83, 053829 (2011).

[31] N. Uribe-Patarroyo, A. Fraine, D. S. Simon, O. Minaeva, and A. V. Sergienko, Phys. Rev. Lett. 110, 043601 (2013).

[32] Y. Gorodetski, K. Y. Bliokh, B. Stein, C. Genet, N. Shitrit, V. Kleiner, E. Hasman, and T. W. Ebbesen, Phys. Rev. Lett. 109, 013901 (2012).

[33] H. Luo, S. Wen, W. Shu, Z. Tang, Y. Zou, and D. Fan, Phys. Rev. A 78, 033805 (2008).

[34] S. Franke-Arnold, G. Gibson, R. W. Boyd, and M. J. Padgett, Science 333, 65 (2011).

[35] J. A. Davis, D. M. Cottrell, J. Campos, M. J. Yzuel, and I. Moreno, Appl. Opt. 38, 5004 (1999).

[36] G. Puentes, N. Hermosa, and J. P. Torres, Phys. Rev. Lett. 109, 040401 (2012).

[37] See Supplemental Material at http://link.aps.org/ supplemental/10.1103/PhysRevLett.112.200401 for more detailed information (Sec. I).

[38] X. F. Qian and J. H. Eberly, Opt. Lett. 36, 4110 (2011).

[39] K. H. Kagalwala, G. Di Giuseppe, A. F. Abouraddy, and B. E. A. Saleh, Nat. Photonics 7, 72 (2012).

[40] See Supplemental Material at http://link.aps.org/ supplemental/10.1103/PhysRevLett.112.200401 for more detailed information (Sec. II).

[41] S. Franke-Arnold, S. M. Barnett, E. Yao, J. Leach, J. Courtial, and M. Padgett, New J. Phys. 6, 103 (2004).

[42] E. Nagali, F. Sciarrino, F. De Martini, L. Marrucci, B. Piccirillo, E. Karimi, and E. Santamato, Phys. Rev. Lett. 103, 013601 (2009).

[43] N. W. M. Ritchie, J. G. Story, and R. G. Hulet, Phys. Rev. Lett. 66, 1107 (1991).

[44] A. Mair, A. Vaziri, G. Weihs, and A. Zeilinger, Nature (London) 412, 313 (2001).

[45] J. Leach, M. J. Padgett, S. M. Barnett, S. Franke-Arnold, and J. Courtial, Phys. Rev. Lett. 88, 257901 (2002).

[46] G. C. G. Berkhout, M. P. J. Lavery, J. Courtial, M. W. Beijersbergen, and M. J. Padgett, Phys. Rev. Lett. 105, 153601 (2010).

[47] M. Mirhosseini, M. Malik, Z. Shi, and R. W. Boyd, Nat. Commun. 4, 2781 (2013).

[48] See Supplemental Material at http://link.aps.org/ supplemental/10.1103/PhysRevLett.112.200401 for more detailed information (Sec. III).

[49] C. Ferrie and J. Combes, Phys. Rev. Lett. 112, 040406 (2014).

[50] G. C. Knee and E. M. Gauger, Phys. Rev. X 4, 011031 (2014).

[51] A. N. Jordan, J. Martínez-Rincón, and J. C. Howell, Phys. Rev. X 4, 011031 (2014). 